\title{
Editorial
}

\section{Computational Algebraic Geometry in String and Gauge Theory}

\author{
Yang-Hui He, ${ }^{1,2,3}$ Philip Candelas, ${ }^{4}$ Amihay Hanany, ${ }^{5}$ \\ Andre Lukas, ${ }^{6}$ and Burt Ovrut ${ }^{7}$
}

${ }^{1}$ Department of Mathematics, City University London, London EC1V 0HB, UK

${ }^{2}$ School of Physics, Nan Kai University, TianJin 300071, China

${ }^{3}$ Merton College, University of Oxford, Oxford OX1 4JD, UK

${ }^{4}$ Mathematical Institute, University of Oxford, 24-29 St Giles', Oxford OX1 3LB, UK

${ }^{5}$ Department of Physics, Imperial College, London SW7 2AZ, UK

${ }^{6}$ Rudolf Peierls Centre for Theoretical Physics, University of Oxford, 1 Keble Road, Oxford OX1 3NP, UK

${ }^{7}$ Department of Physics and Astronomy, University of Pennsylvania, Philadelphia, PA 191046395, USA

Correspondence should be addressed to Yang-Hui He, hey@maths.ox.ac.uk

Received 23 November 2011; Accepted 23 November 2011

Copyright (C 2012 Yang-Hui He et al. This is an open access article distributed under the Creative Commons Attribution License, which permits unrestricted use, distribution, and reproduction in any medium, provided the original work is properly cited.

The last few years have witnessed a rapid development in algebraic geometry, computer algebra, and string and field theory, as well as fruitful cross-fertilization amongst them. The dialogue between geometry and gauge theory is, of course, an old and rich one, leading to tools crucial to both. The introduction of algorithmic and computational algebraic geometry, however, is relatively new and is tremendously facilitated by the rapid progress in hardware, software as well as theory. Applications of once specialized mathematical topics such as Gröbner bases, sheaf cohomology, scheme theory, and Hilbert series are quickly becoming indispensible tools in theoretical physics, from topics ranging from AdS/CFT to string phenomenology, from supersymmetric gauge theory to Calabi-Yau compactifications, and so forth. In this special issue, we have invited many international experts, culminating in 17 papers on related subjects, which we order below alphabetically according to title.

The paper "A simple Introduction to Gröbner basis methods in string phenomenology" by J. Gray is a review on the most important subject in computational and algorithmic algebraic geometry: the Gröbner basis. It illustrates how this can be used in string phenomenology and gives some concrete examples ranging from flux parameter to vacuum spaces. 
The paper "Baryonic symmetries in AdS4/CFT3: an overview" by D. Rodriguez-Gomez is an overview of global baryon-like symmetries in the AdS/CFT context. On the gravity side, these are vector fields in AdS arising from Kaluza-Klein reductions of supergravity $p$ form potentials. The paper focuses on the AdS4/CFT3 case and uses the computation of a noncompact Calabi-Yau fourfold, namely, the cone over the $Q^{111}$, as an explicit example.

The paper "Bell's inequalities, superquantum correlations and string theory" by L. N. Chang et al. is on a more fundamental and philosophical issue in quantum mechanics. It argues that string theory, viewed as a quantum theory with two deformation parameters, the string tension and the string coupling constant, is a superquantum theory which transgresses the usual quantum violations of Bell's inequalities. The norms in the theory generalize from the $\ell^{2}$ norm in functional analysis to an $\ell^{p}$ norm, in application to quantum correlators.

The paper "BPS states, crystals, and matrices" by P. Sułkowski is a review on certain representations of wall-crossing phenomena for toric Calabi-Yau spaces, in relation to free fermions, melting crystal, and matrix models. These have been crucial to the understanding of the BPS spectrum of D-brane theories, in particular D2- and D0-branes bound to a D6-brane. The partition functions which do the counting are intimately related to Donaldson-Thomas invariants and to topological string amplitudes.

The paper "Calabi-Yau threefolds in weighted flag varieties" by M. I. Qureshi and B. Szendrói is on the pure mathematical problem of constructing a large class of Calabi-Yau threefolds as projective varieties. In particular, it considers them as quasilinear sections in weighted flag varieties, generalizing the more familiar cases of the ambient spaces being weighted $\mathbb{P}^{4}$. Certain vector bundles, called tautological orbibundles, on these manifolds are also constructed; these may be of interest to model building in heterotic string theory, when using "general embedding" with such stable bundles.

The paper "Chern-Simons: Fano and Calabi-Yau" by A. Hanany and Y.-H. He returns to the subject of noncompact, toric, Calabi-Yau fourfolds in the context of AdS4/CFT3. It presents the complete classification of smooth toric Fano threefolds, known to the algebraic geometry literature, and performs some preliminary analyses in the context of brane tilings and Chern-Simons theory on M2-branes probing Calabi-Yau fourfold singularities as cones over these Fano threefolds. Emphasis is placed on the fact that these 18 spaces should be as intensely studied as their well-known two complex dimensional counterparts: the del Pezzo surfaces.

The paper "Combinatorics in $N=1$ heterotic vacua" by S.-J. Lee is on constructing bundles over large sets of Calabi-Yau threefolds. It briefly reviews an algorithmic and systematic strategy to explore the landscape of heterotic $E_{8} \times E_{8}$ vacua, in the context of compactifying smooth Calabi-Yau threefolds with vector bundles. The Calabi-Yau threefolds are algebraically realized as hypersurfaces in toric varieties, and a large class of vector bundles is constructed thereon as so-called monad bundles. In the spirit of searching for standard-like heterotic vacua, the focus is on the integer combinatorics of the model-building programme.

The paper "Computational tools for cohomology of toric varieties" by R. Blumenhagen et al. is closely related to the seventh. It addresses a novel computational algorithm for the determination of the dimension of line-bundle-valued cohomology groups on toric varieties. This is clearly useful and can also serve as a first step toward the enormous database of vector bundles on Calabi-Yau hypersurfaces in toric fourfolds. Applications to the computation of chiral massless matter spectra in string compactifications are discussed and the software package "cohom Calg" is advertized. 
The paper "Del Pezzo singularities and SUSY breaking" by D. Malyshev is on del Pezzo singularities in Calabi-Yau threefolds. Singularities of such a type can facilitate dynamical supersymmetry breaking using the Intriligator-Seiberg-Shih technique. An illustrative example is given for the famous quintic manifolds, with its explicit del Pezzo 6 and conifold singularities pointed out. The more general case of complete intersection manifolds is investigated, particularly on the complex deformation of the singularities.

The paper "Discrete Wilson lines in F-theory" by V. Braun is on elliptically fibered CalabiYau fourfolds to F-theory constructions. Recent works by Heckmann, Vafa et al. have realized the GUT group from sevenbranes wrapping contractible del Pezzo surfaces in the fourfold. However, this makes breaking to the Standard Model group difficult since the del Pezzo surfaces have trivial fundamental group. The paper shows how one may use non-trivial cycles, such as the Enriquez surface, and use Wilson lines to break the GUT gauge group.

The paper "Non-supersymmetric CS-matter theories with known AdS duals" by D. Forcella and A. Zaffaroni is related to the second and sixth and studies M2-branes probing CalabiYau fourfold cones. The dual supergravity solutions of Freund-Rubin type are found while the world-volume field theories are stable and non-supersymmetric. Careful analyses for the theory associated to the cone over the quotient $S^{7} / \mathbb{Z}_{k}$ are carried out, giving the Kaluza-Klein spectrum and candidate dual gauge theory.

The paper "Numerical polynomial homotopy continuation method and string vacua" by D. Mehta forms a nice contrast with the first and focuses on algebraic geometry without using the Gröbner basis which is known to be quite expensive computationally. Instead, it shows how one may sometimes obtain relevant information, exemplified by various models taken from string and M-theory as well as lattice gauge theory, by using numerical algebraic geometry. Emphasis is paid on the so-called homotopy continuation method.

The paper "On $R^{4}$ terms and MHV amplitudes in $N=5,6$ supergravity vacua of type II superstrings" by M. Bianchi is on $N=5$ and 6 supergravity vacua of type II superstring theory. It computes the one-loop threshold corrections to the 4th power of curvature terms as well as nonperturbative corrections of D-brane instantons. The generating functions for maximally helicity violating amplitudes at tree level are also derived.

The paper "On the minimal length uncertainty relation and the foundations of string theory" by L. N. Chang et al. echoes the third and reviews the work on minimal length uncertainty as suggested by string theory, in particular what happens to quantum mechanics when a fundamental length scale such as the string length is introduced. The implications of this to the vacuum energy and to a dynamical energy-momentum space are discussed.

The paper "Polynomial roots and Calabi-Yau geometries" by Y.-H. He is a numerical and algorithmic experiment, inspired by the fractal nature of the roots of large sets of polynomials with certain constrained coefficients. It looks at the space of Poincaré polynomials (a generalization of Euler number) of all known compact Calabi-Yau threefolds and fourfolds and studies the structure exhibited by their conglomerate roots.

The paper "The expanding zoo of Calabi-Yau threefolds" by R. Davies is on the algorithmic search for new Calabi-Yau threefolds with special properties. It reviews the recent constructions of the manifolds of small Hodge number and/or nontrivial fundamental group, which are useful to heterotic model building. The techniques used are topological transitions and quotienting from known manifold by discrete group actions. A first example of a CalabiYau threefold with the fundamental group $S_{3}$ is given. 
The paper "Toric methods in F-theory model building" by J. Knapp and M. Kreuzer is related to the seventh, eighth, and the tenth. It reviews how the construction of Ftheory models calls for large databases of Calabi-Yau fourfolds which are elliptically fibered. Furthermore, one needs to look for a divisor which is a nonnegatively curved threefold serving as the base and, further still, a divior inside the base which is a del Pezzo surface. The paper shows how one may do this search algorithmically and explicitly demonstrates this with the powerful computer package "PALP."

Yang-Hui He

Philip Candelas

Amihay Hanany

Andre Lukas

Burt Ovrut 

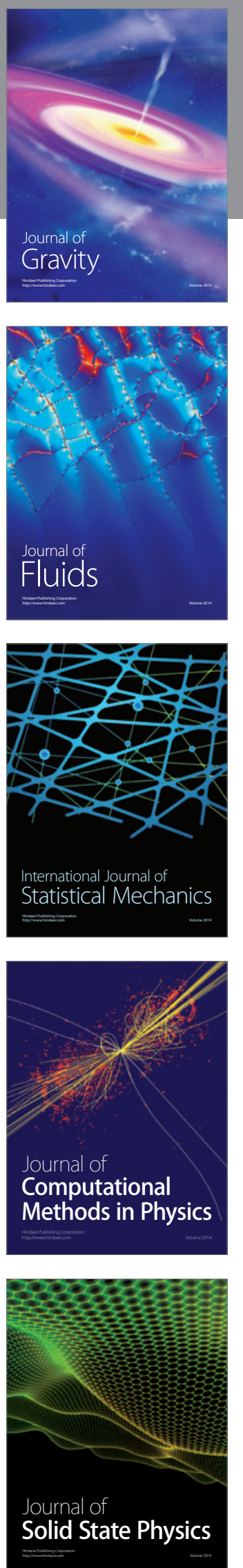

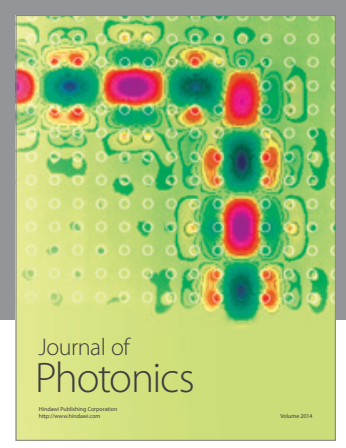

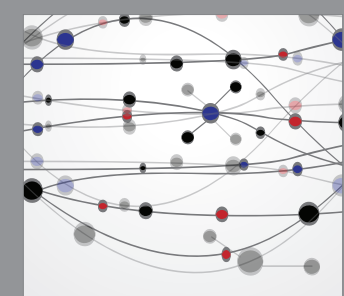

The Scientific World Journal
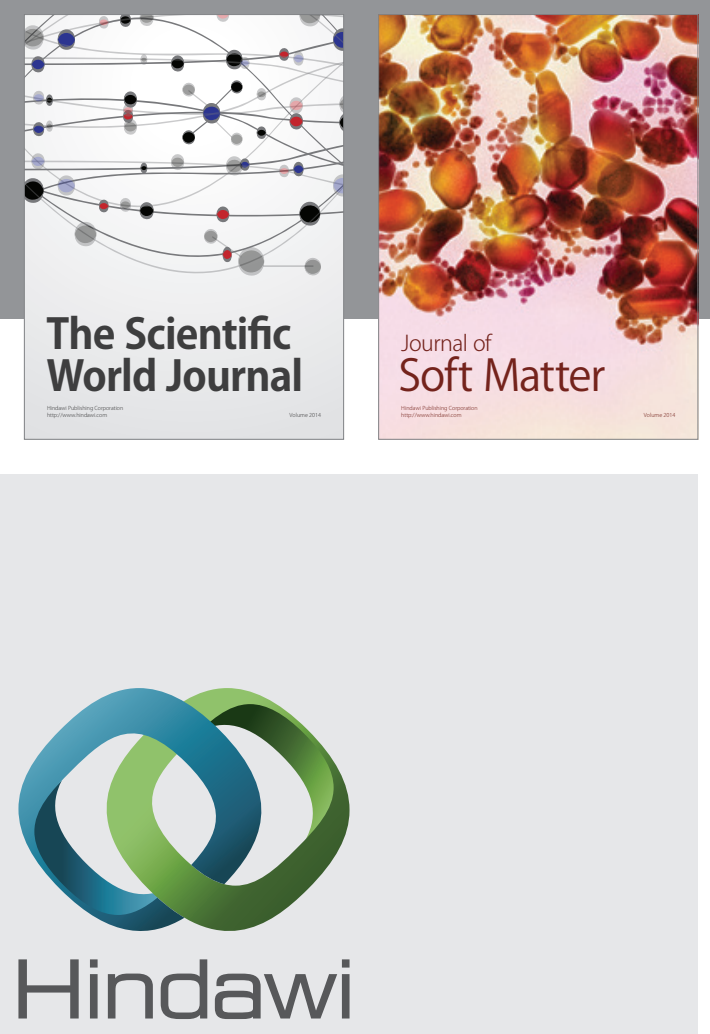

Submit your manuscripts at

http://www.hindawi.com
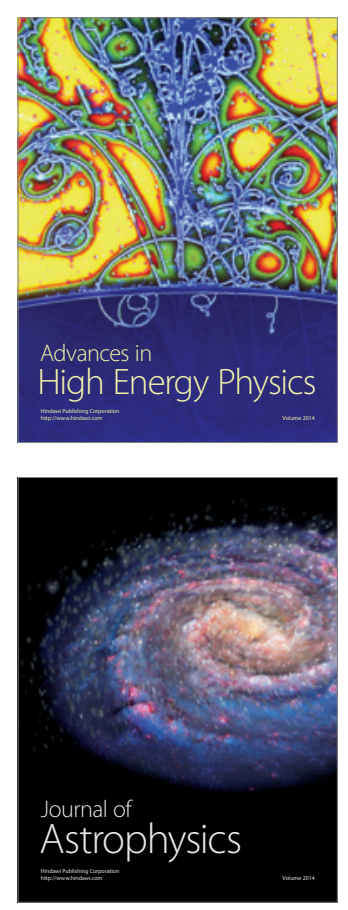
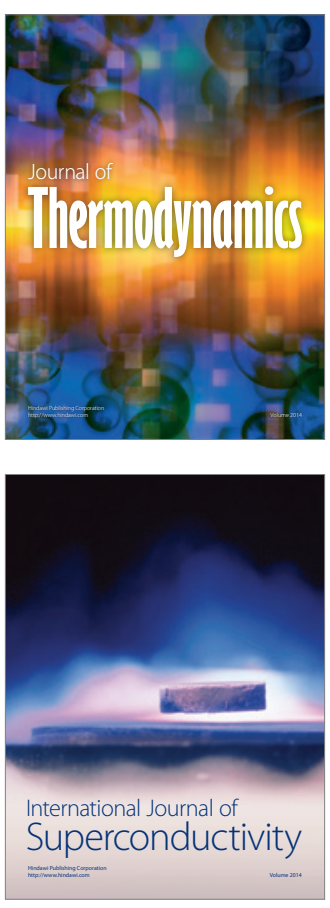
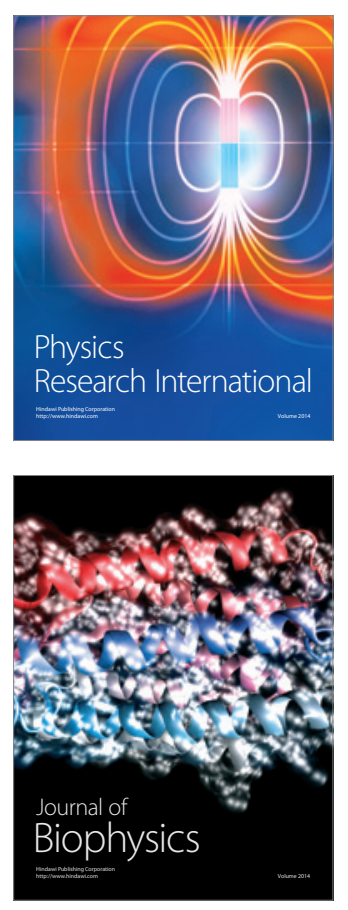
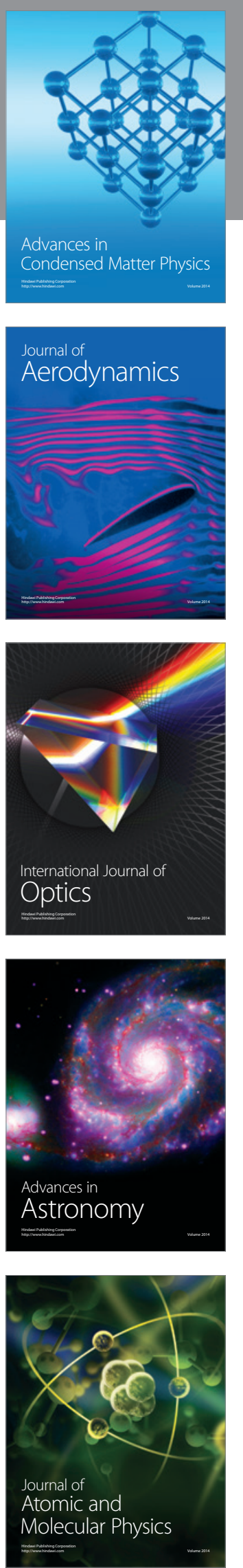